Article

\title{
Extended Mizoguchi-Takahashi Type Fixed Point Theorems and Their Application
}

\author{
Babak Mohammadi ${ }^{1}$, Vahid Parvaneh ${ }^{2, *}$, Hassen Aydi ${ }^{3,4}$ (D) and Hüseyin Işı1 ${ }^{5,6, *(D)}$ \\ 1 Department of Mathematics, Marand Branch, Islamic Azad University, Marand, Iran \\ Department of Mathematics, Gilan-E-Gharb Branch, Islamic Azad University, Gilan-E-Gharb, Iran \\ 3 Institut Supérieur d'Informatique et des Techniques de Communication, Université de Sousse, H. Sousse \\ 4000, Tunisia \\ 4 China Medical University Hospital, China Medical University, Taichung 40402, Taiwan \\ 5 Nonlinear Analysis Research Group, Ton Duc Thang University, Ho Chi Minh City 700000, Vietnam \\ 6 Faculty of Mathematics and Statistics, Ton Duc Thang University, Ho Chi Minh City 700000, Vietnam \\ * Correspondence: vahid.parvaneh@kiau.ac.ir (V.P.); huseyin.isik@tdtu.edu.vn (H.I.)
}

Received: 10 May 2019; Accepted: 24 June 2019; Published: 27 June 2019

\begin{abstract}
The aim of this work is to extend the Mizoguchi-Takahashi fixed point result motivated by the approach of Wardowski (2012) and provide some related fixed point results in (ordered) metric spaces. An example is given to support the main results. Moreover, we provide an application on nonlinear differential equations.
\end{abstract}

Keywords: metric space; partially ordered set; fixed point; contraction

\section{Introduction}

Ran and Reurings [1] investigated fixed point results on partially ordered sets. This approach has been recently considered by many authors, see [2-13]. Zaslavski [14] proved two fixed point results for a class of contraction type mappings on a closed subset of a complete metric space. Given a metric space $(X, d)$. Following [15], denote by $C B(X)$ (respectively, $K(X))$ the class of non-empty closed bounded (respectively, non-empty compact) subsets of $X$. Let $H$ be the Hausdorff-Pompeiu metric on $C B(X)$ induced by the metric $d$. It is given as

$$
H\left(\mathrm{Y}_{1}, \mathrm{Y}_{2}\right)=\max \left\{\sup _{\varsigma_{1} \in \mathrm{Y}_{1}} d\left(\varsigma_{1}, \mathrm{Y}_{2}\right), \sup _{\varsigma_{2} \in \mathrm{Y}_{2}} d\left(\varsigma_{2}, \mathrm{Y}_{1}\right)\right\},
$$

for all $Y_{1}, Y_{2} \in C B(X)$.

An element $\theta \in X$ is said to be a fixed point of a multi-valued mapping $T$ if $\theta \in T \theta$. For fixed point results dealing with the multi-valued case, see [16-19].

Let $(X, d)$ be a complete metric space and $T: X \longrightarrow K(X)$ be a multi-valued mapping such that

$$
H(T \omega, T \Omega) \leq \alpha(d(\omega, \Omega)) d(\omega, \Omega)
$$

for all $\omega \neq \Omega \in X$, where $\alpha:(0, \infty) \longrightarrow[0,1)$ is a mapping such that for each $t \in[0, \infty)$, $\lim \sup _{r \rightarrow t^{+}} \alpha(r)<1$, then $T$ has a fixed point, see [20].

Reich [20] stated a question of whether $K(X)$ can be replaced by $C B(X)$ in the above result. Mizoguchi and Takahashi [21] gave a positive answer to the conjecture of Reich.

Theorem 1. Let $(X, d)$ be a complete metric space and let $T: X \rightarrow C B(X)$ be a multi-valued mapping such that

$$
H(T \omega, T \Omega) \leq \alpha(d(\omega, \Omega)) d(\omega, \Omega),
$$


for all $\omega, \Omega \in X$, where $\alpha:(0, \infty) \rightarrow[0,1)$ verifies $\lim \sup \alpha(t)<1$, for each $r \geq 0$. Then $T$ has a fixed point $([21])$.

Denote by $\Psi$ the family of functions $\psi:[0, \infty) \longrightarrow[0, \infty)$ so that

1. $\psi(s)=0 \Leftrightarrow s=0$;

2. $\psi$ is nondecreasing and lower semi-continuous;

3. $\limsup _{\kappa \rightarrow 0^{+}} \frac{\kappa}{\psi(\kappa)}<\infty$.

Consider, $(H)$ : for any increasing sequence $\left\{\varsigma_{n}\right\}$ in $X$ with $\varsigma_{n} \rightarrow x$ as $n \rightarrow \infty$, we have $\varsigma_{n} \preceq x$ for each $n \geq 0$. Gordji and Ramezani [22] considered a variant of Theorem 1 for single-valued mappings. Given a partial order $\preceq$ on a non-empty set $X$, we say that $\omega$ and $\Omega$ in $X$ are comparable if $\omega \preceq \Omega$ or $\Omega \preceq \omega$.

Theorem 2. Let $(X, d, \preceq)$ be a complete partially ordered metric space. Let $f: X \longrightarrow X$ be an increasing mapping so that there is $\varsigma_{0} \in X$ with $\varsigma_{0} \preceq f\left(\varsigma_{0}\right)$ ([22]). Suppose there is $\psi \in \Psi$ so that

$$
\psi(d(f \omega, f \Omega)) \leq \alpha(\psi(d(\omega, \Omega))) \psi(d(\omega, \Omega))
$$

for all comparable $\omega, \Omega \in X$, where $\alpha:[0, \infty) \longrightarrow(0,1)$ verifies $\lim \sup _{s \rightarrow t^{+}} \alpha(s)<1$, for any $t \geq 0$. If either $f$ is continuous, or $(H)$ holds, then there is a fixed point of $f$.

Definition 1 ([23]). Given a self-mapping $f$ on $X$ and $\alpha: X^{2} \rightarrow[0, \infty)$. Such $f$ is triangular $\alpha$-admissible if (T1) $\alpha(\omega, \Omega) \geq 1 \quad$ implies $\quad \alpha(f \omega, f \Omega) \geq 1, \omega, \Omega \in X$,

(T2) $\left\{\begin{array}{l}\alpha(\omega, \zeta) \geq 1 \\ \alpha(\zeta, \Omega) \geq 1\end{array} \quad\right.$ implies $\quad \alpha(\omega, \Omega) \geq 1, \quad \omega, \Omega, \zeta \in X$.

Example 1 ([23]). Let $X=\mathbb{R}$. Take $f \Omega=\sqrt[3]{\Omega}$ and $\alpha(\omega, \Omega)=e^{\omega-\Omega}$. Here, $f$ is a triangular $\alpha$-admissible mapping.

Lemma 1 ([23]). Let $f$ be a triangular $\alpha$-admissible self-mapping on a non-empty set $X$. Assume that there is $\varsigma_{0} \in X$ so that $\alpha\left(\varsigma_{0}, f \varsigma_{0}\right) \geq 1$. Take $\left\{\varsigma_{n}\right\}$ as $\varsigma_{n}=f^{n} \varsigma_{0}$, then

$$
\alpha\left(\varsigma_{p}, \varsigma_{q}\right) \geq 1 \text { for all } p, q \in \mathbb{N} \text { with } p<q
$$

In this paper, we obtain some fixed point theorems for triangular $\alpha$-admissible Mizoguchi-Takahashi type contractions. We also derive variant related theorems for nondecreasing mappings in ordered metric spaces. Moreover, we provide an application for nonlinear differential equations. These results generalize several comparable ones in the literature.

\section{Main Results}

Denote by $\Phi$ the set of the functions $\beta:(0, \infty) \longrightarrow[0,1)$ such that $\lim \sup _{\omega \rightarrow t^{+}} \beta(\omega)<1$, for any $t \geq 0$.

Denote by $\mathcal{F}$ the set of all functions $F:(0, \infty) \rightarrow \mathbb{R}$ so that:

$\left(F_{1}\right) F$ is strictly increasing and continuous;

$\left(F_{2}\right) F(t)=0 \quad \Leftrightarrow \quad t=1$.

The functions $\ln (t)$ and $-\frac{1}{\sqrt{t}}+1$ are elements of $\mathcal{F}$. 
Denote by $\Lambda$ the family of functions $\psi:[0, \infty) \longrightarrow[0, \infty)$ so that

1. $\psi(s)=0 \Leftrightarrow s=0$;

2. $\psi$ is nondecreasing and continuous.

For $\omega, \Omega \in X$, consider

$$
M(\omega, \Omega)=\max \{d(\omega, \Omega), d(\omega, f \omega), d(\Omega, f \omega)\},
$$

where $d$ is a metric on $X$.

Take: $(K)$ : Whenever $\left\{\varsigma_{n}\right\}$ is each sequence in $X$ so that $\alpha\left(\varsigma_{n}, \varsigma_{n+1}\right) \geq 1$ for each integer $n \geq 0$ and $\varsigma_{n} \rightarrow x$ as $n \rightarrow+\infty$, we have $\alpha\left(\varsigma_{n}, x\right) \geq 1$ for each $n \geq 0$.

Now, we give the main result of this study.

Theorem 3. Let $f$ be a self-mapping on a complete metric space $(X, d)$. Suppose that there is a function $\alpha: X^{2} \rightarrow[0, \infty)$ satisfying

$$
F(\alpha(\omega, \Omega) \psi(d(f \omega, f \Omega))) \leq F(\beta(\psi(d(\omega, \Omega))))+F(\psi(M(\omega, \Omega)))
$$

for all $\omega, \Omega \in X$ with $f \omega \neq f \Omega$, where $F \in \mathcal{F}, \beta \in \Phi$ and $\psi \in \Lambda$. Assume that $f$ is triangular $\alpha$-admissible and there is $\varsigma_{0} \in X$ so that $\alpha\left(\varsigma_{0}, f \varsigma_{0}\right) \geq 1$. Then $f$ has a fixed point if,

(a) either $f$ is continuous, or;

(b) (K) holds.

Moreover, if for any two fixed points $\omega, \Omega$ of $f$, we have $\alpha(\omega, \Omega) \geq 1$, then such a fixed point is unique.

Proof. Let $\varsigma_{0} \in X$ be such that $\alpha\left(\varsigma_{0}, f \varsigma_{0}\right) \geq 1$. Define $\left\{\varsigma_{n}\right\}$ as $\varsigma_{n+1}=f \varsigma_{n}$ for each $n \geq 0$.

As $\alpha\left(\varsigma_{0}, \varsigma_{1}\right)=\alpha\left(\varsigma_{0}, f \varsigma_{0}\right) \geq 1$, then using the $\alpha$-admissibility, one writes $\alpha\left(\varsigma_{1}, \varsigma_{2}\right)=\alpha\left(f \varsigma_{0}, f \varsigma_{1}\right) \geq 1$. Continuing in same direction, we have $\alpha\left(\varsigma_{n}, \varsigma_{n+1}\right) \geq 1$ for any $n \geq 0$.

If $\varsigma_{n}=\varsigma_{n+1}$ for some $n \geq 0$, then the proof is done. Now, assume that $\varsigma_{n} \neq \varsigma_{n+1}$ for each $n \geq 0$, that is,

$$
d\left(\varsigma_{n}, \varsigma_{n+1}\right)>0,
$$

for each $n \geq 0$. Define $\delta_{n}:=d\left(\varsigma_{n}, \varsigma_{n+1}\right)$. In view of (4), we obtain that

$$
\begin{aligned}
F\left(\psi\left(d\left(\varsigma_{n+1}, \varsigma_{n+2}\right)\right)\right) & \leq F\left(\alpha\left(\varsigma_{n}, \varsigma_{n+1}\right) \psi\left(d\left(\varsigma_{n+1}, \varsigma_{n+2}\right)\right)\right) \\
& =F\left(\alpha\left(\varsigma_{n}, \varsigma_{n+1}\right) \psi\left(d\left(f \varsigma_{n}, f \varsigma_{n+1}\right)\right)\right) \\
& \leq F\left(\beta\left(\psi\left(d\left(\varsigma_{n}, \varsigma_{n+1}\right)\right)\right)\right)+F\left(\psi\left(M\left(\varsigma_{n}, \varsigma_{n+1}\right)\right)\right)
\end{aligned}
$$

where

$$
M\left(\varsigma_{n}, \varsigma_{n+1}\right)=\max \left\{d\left(\varsigma_{n}, \varsigma_{n+1}\right), d\left(\varsigma_{n}, f \varsigma_{n}\right), d\left(\varsigma_{n+1}, f \varsigma_{n}\right)\right\}=d\left(\varsigma_{n}, \varsigma_{n+1}\right) .
$$

Therefore,

$$
F\left(\psi\left(d\left(\varsigma_{n+1}, \varsigma_{n+2}\right)\right)\right) \leq F\left(\beta\left(\psi\left(d\left(\varsigma_{n}, \varsigma_{n+1}\right)\right)\right)\right)+F\left(\psi\left(d\left(\varsigma_{n}, \varsigma_{n+1}\right)\right)\right)
$$

for each $n \geq 0$. Put $t_{n}:=\psi\left(d\left(\varsigma_{n}, \varsigma_{n+1}\right)\right)$. Using (6), we have

$$
F\left(t_{n+1}\right) \leq F\left(\beta\left(t_{n}\right)\right)+F\left(t_{n}\right), \text { for each } n \geq 0 .
$$

Since $\beta\left(t_{n}\right)<1$ and $F$ is strictly increasing, we get $F\left(\beta\left(t_{n}\right)\right)<F(1)=0$. Therefore, from (7), we have

$$
F\left(t_{n+1}\right) \leq F\left(\beta\left(t_{n}\right)\right)+F\left(t_{n}\right)<F\left(t_{n}\right), \text { for each } n \geq 0 .
$$


Since $F$ is strictly increasing, we get $t_{n+1}<t_{n}$ and so there is $r \geq 0$ such that, $t_{n} \rightarrow r^{+}$. Now, we show that $r=0$. Suppose to the contrary $r>0$. Passing to the limit throw (8), $F(r) \leq F\left(\limsup _{n \rightarrow \infty}\left(\beta\left(t_{n}\right)\right)\right)+F(r)<F(r)$, which is a contradiction. Hence $\lim _{n \rightarrow \infty} t_{n}=r=0$. Since $\left\{\psi\left(d\left(\varsigma_{n}, \varsigma_{n+1}\right)\right)\right\}$ is decreasing and $\psi$ is increasing, so $\left.\left\{d\left(\varsigma_{n}, \varsigma_{n+1}\right)\right)\right\}$ is decreasing. Then there is $u \geq 0$ so that $\left\{d\left(\varsigma_{n}, \varsigma_{n+1}\right)\right\}$ converges to $u$. Since $\psi$ is continuous, one writes

$$
\psi(u)=\lim _{n \longrightarrow \infty} \psi\left(d\left(\varsigma_{n}, \varsigma_{n+1}\right)\right)=r=0 .
$$

Therefore, $u=0$. We claim that $\left\{\zeta_{n}\right\}$ is a Cauchy sequence. If $\left\{\zeta_{n}\right\}$ is not Cauchy, then there are $\varepsilon>0$ and subsequences $\left\{\zeta_{m_{i}}\right\}$ and $\left\{\varsigma_{n_{i}}\right\}$ of $\left\{\varsigma_{n}\right\}$ so that

$$
n_{i}>m_{i}>i, d\left(\varsigma_{m_{i}}, \varsigma_{n_{i}}\right) \geq \varepsilon
$$

and

$$
d\left(\varsigma_{m_{i}}, \varsigma_{n_{i}-1}\right)<\varepsilon
$$

Using (10), we get

$$
\varepsilon \leq d\left(\varsigma_{m_{i}}, \varsigma_{n_{i}}\right) \leq d\left(\varsigma_{m_{i}}, \varsigma_{n_{i}-1}\right)+d\left(\varsigma_{n_{i}-1}, \varsigma_{n_{i}}\right)<\varepsilon+d\left(\varsigma_{n_{i}-1}, \varsigma_{n_{i}}\right) .
$$

As $i \rightarrow \infty$, we find

$$
\lim _{i \rightarrow \infty} d\left(\varsigma_{m_{i}}, \varsigma_{n_{i}}\right)=\varepsilon
$$

Also, we have

$$
\begin{aligned}
d\left(\varsigma_{m_{i}}, \varsigma_{n_{i}}\right)-d\left(\varsigma_{m_{i}}, \varsigma_{m_{i}+1}\right)-d\left(\varsigma_{n_{i}}, \varsigma_{n_{i}+1}\right) & \leq d\left(\varsigma_{m_{i}+1}, \varsigma_{n_{i}+1}\right) \\
& \leq d\left(\varsigma_{m_{i}}, \varsigma_{m_{i}+1}\right)+d\left(\varsigma_{m_{i}}, \varsigma_{n_{i}}\right)+d\left(\varsigma_{n_{i}}, \varsigma_{n_{i}+1}\right) .
\end{aligned}
$$

As $i \rightarrow \infty$, we find

$$
\lim _{i \rightarrow \infty} d\left(\varsigma_{m_{i}+1}, \varsigma_{n_{i}+1}\right)=\varepsilon .
$$

The triangular $\alpha$-admissibility yields that $\alpha\left(\varsigma_{m_{i}}, \varsigma_{n_{i}}\right) \geq 1$. By (4), we find

$$
\begin{aligned}
F\left(\psi\left(d\left(\varsigma_{m_{i}+1}, \varsigma_{n_{i}+1}\right)\right)\right) & \leq F\left(\alpha\left(\varsigma_{m_{i}}, \varsigma_{n_{i}}\right) \psi\left(d\left(\varsigma_{m_{i}+1}, \varsigma_{n_{i}+1}\right)\right)\right) \\
& \leq F\left(\beta\left(\psi\left(d\left(\varsigma_{m_{i}}, \varsigma_{n_{i}}\right)\right)\right)+F\left(\psi\left(M\left(\varsigma_{m_{i}}, \varsigma_{n_{i}}\right)\right) .\right.\right.
\end{aligned}
$$

On the other hand,

$$
\begin{aligned}
& d\left(\varsigma_{m_{i}}, \varsigma_{n_{i}}\right) \leq M\left(\varsigma_{m_{i}}, \varsigma_{n_{i}}\right) \\
& =\max \left\{d\left(\varsigma_{m_{i}}, \varsigma_{n_{i}}\right), d\left(\varsigma_{m_{i}}, \varsigma_{m_{i}+1}\right), d\left(\varsigma_{n_{i}}, \varsigma_{m_{i}+1}\right)\right\} \\
& \leq \max \left\{d\left(\varsigma_{m_{i}}, \varsigma_{n_{i}}\right), d\left(\varsigma_{m_{i}}, \varsigma_{m_{i}+1}\right), d\left(\varsigma_{m_{i}}, \varsigma_{n_{i}}\right)+d\left(\varsigma_{m_{i}}, \varsigma_{m_{i}+1}\right)\right\}
\end{aligned}
$$

As $i \rightarrow \infty$, we find

$$
\lim _{i \rightarrow \infty} M\left(\varsigma_{m_{i}}, \varsigma_{n_{i}}\right)=\varepsilon
$$

Taking the limit on both sides of (15), we have

$$
F(\psi(\varepsilon)) \leq F\left(\lim \sup _{i \rightarrow \infty} \beta\left(\psi\left(d\left(\varsigma_{m_{i}}, \varsigma_{n_{i}}\right)\right)\right)+F(\psi(\varepsilon)) .\right.
$$

Since $d\left(\varsigma_{m_{i}}, \varsigma_{n_{i}}\right) \rightarrow \varepsilon^{+}$and $\psi$ is increasing, thus $\psi\left(d\left(\varsigma_{m_{i}}, \varsigma_{n_{i}}\right)\right) \rightarrow \psi(\varepsilon)^{+}$. So $\limsup _{i \rightarrow \infty} \beta\left(\psi\left(d\left(\varsigma_{m_{i}}, \varsigma_{n_{i}}\right)\right)<1\right.$. Therefore, $F\left(\lim \sup _{i \rightarrow \infty} \beta\left(\psi\left(d\left(\varsigma_{m_{i}}, \varsigma_{n_{i}}\right)\right)\right)<0\right.$. Thus (16) leads 
to $F(\psi(\varepsilon))<F(\psi(\varepsilon))$, a contradiction.

Thus, $\left\{s_{n}\right\}$ is a Cauchy sequence in the complete metric space $(X, d)$, hence there is $x \in X$ so that

$$
\lim _{n \rightarrow \infty} \varsigma_{n}=x
$$

Finally, we claim that $f x=x$.

If $f$ is a continuous function, then obviously, $f x=x$.

Let condition $(b)$ hold. To show that $f x=x$, we have two cases:

Case 1: There is $N \in \mathbb{N}$ so that $f \varsigma_{n} \neq f x$ for each $n \geq N$.

Case 2: There is a subsequence $\left\{\zeta_{n_{i}}\right\}$ of $\left\{\zeta_{n}\right\}$ so that $f \varsigma_{n_{i}}=f x$ for each $i \geq 0$.

In Case 1, if $d(x, f x) \neq 0$, we have

$$
\begin{aligned}
F\left(\psi\left(d\left(\varsigma_{n+1}, f x\right)\right)\right) & =F\left(\psi\left(d\left(f \varsigma_{n}, f x\right)\right)\right) \\
& \leq F\left(\beta\left(\psi\left(d\left(\varsigma_{n}, x\right)\right)\right)+F\left(\psi\left(M\left(\varsigma_{n}, x\right)\right)\right)\right. \\
& <F\left(\psi\left(M\left(\varsigma_{n}, x\right)\right)\right) .
\end{aligned}
$$

This gives us

$$
\psi\left(d\left(\varsigma_{n+1}, f x\right)\right)<\psi\left(M\left(\varsigma_{n}, x\right)\right) \text { for each } n \geq N
$$

Also

$$
\begin{aligned}
\lim _{n \longrightarrow \infty} M\left(\varsigma_{n}, x\right) & =\lim _{n \longrightarrow \infty} \max \left\{d\left(\varsigma_{n}, x\right), d\left(\varsigma_{n}, \varsigma_{n+1}\right), d\left(\varsigma_{n+1}, x\right)\right\} \\
& =0 .
\end{aligned}
$$

Passing to the limit using (19), we obtain $\psi(d(x, f x)) \leq 0$. Hence, $d(x, f x)=0$.

In Case 2,

$$
d(x, f x)=\lim _{n \rightarrow \infty} d\left(\varsigma_{n+1}, f x\right)=\lim _{n \rightarrow \infty} d\left(f_{\varsigma_{n}}, f x\right)=0 .
$$

We deduce that $f x=x$. To show the uniqueness of the fixed point, suppose that $\omega, \Omega$ are two distinct fixed points of $f$. By assumption, we have $\alpha(\omega, \Omega) \geq 1$. Using (4), we have

$$
\begin{aligned}
F(\psi(d(\omega, \Omega))) & =F(\psi(d(f \omega, f \Omega))) \leq F(\alpha(\omega, \Omega) \psi(d(f \omega, f \Omega))) \\
& \leq F(\beta(\psi(d(\omega, \Omega))))+F(\psi(M(\omega, \Omega))) \\
& =F(\beta(\psi(d(\omega, \Omega))))+F(\psi(d(\omega, \Omega))) .
\end{aligned}
$$

From the above inequality, we get $F(\beta(\psi(d(\omega, \Omega)))) \geq 0$, which implies that $\beta(\psi(d(\omega, \Omega))) \geq 1$. It is a contradiction. Thus, $\omega=\Omega$.

Let $(X, \preceq)$ be an ordered space. A subset $W$ of $X$ is called well ordered, whenever any two elements $\omega, \Omega \in X$ are comparable, that is, $\omega \leq \Omega$ or $\Omega \leq \omega$. The following Theorem is a straightforward result of Theorem 3 in ordered metric spaces.

Theorem 4. Let $(X, d, \preceq)$ be an ordered complete metric space. Let $f: X \rightarrow X$ be such that

$$
F(\psi(d(f \omega, f \Omega))) \leq F(\beta(\psi(d(\omega, \Omega))))+F(\psi(M(\omega, \Omega)))
$$

for all $\omega, \Omega \in X$ with $\omega \preceq \Omega$ and $f \omega \neq f \Omega$, where $F \in \mathcal{F}, \beta \in \Phi$ and $\psi \in \Lambda$. Then $f$ has a fixed point if

(i) $f$ is nondecreasing with respect to $\preceq$;

(ii) there is $\varsigma_{0} \in X$ so that $\varsigma_{0} \preceq f \varsigma_{0}$;

(iii) either $f$ is continuous, or

(iii) $^{\prime}(H)$ holds. 
Moreover, if Fix(f) (the set of fixed points of $f$ ) is well ordered, then such a fixed point is unique.

Taking $F(t)=\ln (t)$ in Theorem 3, we have

Corollary 1. Let $f$ be a self-mapping on a complete metric space $(X, d)$. Given $\alpha: X^{2} \rightarrow[0, \infty)$, let

(i) $f$ is triangular $\alpha$-admissible;

(ii) for all $\omega, \Omega \in X$ such that $d(f \omega, f \Omega)>0$, we have

$$
\alpha(\omega, \Omega) \psi(d(f \omega, f \Omega)) \leq \beta(\psi(d(\omega, \Omega))) \psi(M(\omega, \Omega))
$$

where $\beta:[0, \infty) \rightarrow[0,1)$ is the Mizogochi-Takahashi function and $\psi \in \Lambda$;

(iii) there is $\varsigma_{0} \in X$ so that $\alpha\left(\varsigma_{0}, f \varsigma_{0}\right) \geq 1$;

(iv) either $f$ is continuous, or $(K)$ holds.

Then $f$ has a fixed point. Moreover, such a fixed point is unique provided that $\alpha(\omega, \Omega) \geq 1$ for all $\omega, \Omega \in$ $\operatorname{Fix}(f)$.

Proof. Taking $\ln$ in both sides of (21), we obtain

$$
\ln (\alpha(\omega, \Omega) \psi(d(f \omega, f \Omega))) \leq \ln (\beta(\psi(d(\omega, \Omega))))+\ln (\psi(M(\omega, \Omega))) .
$$

Putting $F(t)=\ln (t)$ in above inequality, we have (4). Thus, the result is followed from Theorem 3.

Corollary 2. Let $(X, \preceq)$ be a partially ordered set and suppose that there exists a metric $d$ on $X$ such that $(X, d)$ is complete. Let $f: X \longrightarrow X$ be an increasing mapping such that there is $\varsigma_{0} \in X$ with $\varsigma_{0} \preceq f \varsigma_{0}$. Suppose that there are $\psi \in \Psi$ and $\beta \in \Phi$ such that

$$
\psi(d(f \omega, f \Omega)) \leq \beta(\psi(d(\omega, \Omega))) \psi(M(\omega, \Omega))
$$

for all comparable $\omega, \Omega \in X$, where $\beta:[0, \infty) \longrightarrow[0,1)$ is such that $\lim \sup _{s \rightarrow t^{+}} \beta(s)<1$, for each $t \geq 0$. Assume that either $f$ is continuous, or $(H)$ holds. Then $f$ has a fixed point. Moreover, if Fix $(f)$ is well ordered, then such a fixed point is unique.

Proof. Taking $\ln$ in both sides of (23), we obtain

$$
\ln (\psi(d(f \omega, f \Omega))) \leq \ln (\beta(\psi(d(\omega, \Omega))))+\ln (\psi(M(\omega, \Omega)))
$$

for all comparable $\omega, \Omega \in X$. Putting $F(t)=\ln (t)$ in above inequality, we have (20). Thus the result is followed from Theorem 4 .

Remark 1. Theorems 3 and 4 are generalizations of the main result in [22] and the Mizogochi-Takahashi result for self-mappings. In the following example, we show that these generalizations are real.

Example 2. Let $X=\{1,2,3\}$. We endow $X$ with the metric d defined by $d(1,2)=\frac{1}{2}, d(2,3)=\frac{1}{3}, d(1,3)=\frac{5}{6}$. Consider

$$
\alpha(\omega, \Omega)= \begin{cases}1, & \text { if }(\omega, \Omega) \in\{(1,1),(2,2),(3,3),(1,3),(3,2),(1,2)\}, \\ 0, & \text { otherwise. }\end{cases}
$$

Also, take $f: X \rightarrow X$ as

$$
f=\left(\begin{array}{lll}
1 & 2 & 3 \\
3 & 2 & 2
\end{array}\right)
$$


Here, $f$ is triangular $\alpha$-admissible. For $\varsigma_{0}=1$, we have $f \varsigma_{0}=3$ and $\alpha\left(\varsigma_{0}, f \varsigma_{0}\right)=\alpha(1,3)=1$. Choose $F(t)=-\frac{1}{\sqrt{t}}+1, \beta(t)=e^{-t}$ and $\psi(t)=t$. Let $\omega, \Omega \in X$ such that $\alpha(\omega, \Omega) \geq 1$ and $d(f \omega, f \Omega)>0$. Here, $(\omega, \Omega) \in\{(1,2),(1,3)\}$.

If $(\omega, \Omega)=(1,2)$, then $d(f 1, f 2)=d(3,2)=\frac{1}{3}$. Now,

$$
\begin{aligned}
F(d(f 1, f 2)) & =\frac{-1}{\sqrt{\frac{1}{3}}}+1=-0.732 \leq-0.698=-0.284-0.414 \\
& =\left(\frac{-1}{\sqrt{e^{-\frac{1}{2}}}}+1\right)+\left(\frac{-1}{\sqrt{\frac{1}{2}}}+1\right) \\
& =F(\beta(\psi(d(\omega, \Omega))))+F(\psi(d(\omega, \Omega))) \\
& \leq F(\beta(\psi(d(\omega, \Omega))))+F(\psi(M(\omega, \Omega)))
\end{aligned}
$$

If $(\omega, \Omega)=(1,3)$, then $d(f 1, f 2)=d(3,2)=\frac{1}{3}$. Here,

$$
\begin{aligned}
F(\psi(d(f 1, f 2))) & =\frac{-1}{\sqrt{\frac{1}{3}}}+1=-0.732 \leq-0.673=-0.578-0.0954 \\
& =\left(\frac{-1}{\sqrt{e^{-\frac{5}{6}}}}+1\right)+\left(\frac{-1}{\sqrt{\frac{5}{6}}}+1\right) \\
& =F(\beta(\psi(d(\omega, \Omega))))+F(\psi(d(\omega, \Omega))) \\
& \leq F(\beta(\psi(d(\omega, \Omega))))+F(\psi(M(\omega, \Omega))) .
\end{aligned}
$$

Therefore, (4) holds for all $\omega, \Omega$ with $\alpha(\omega, \Omega) \geq 1$ and $d(f \omega, f \Omega)>0$. We see that all of the conditions of Theorem 3 are satisfied, so $f$ has a unique fixed point, which is, $r=2$. Note that

$$
\psi(d(f 1, f 2))=d(3,2)=\frac{1}{3}>0.303=\left(\frac{1}{2}\right)\left(e^{-\frac{1}{2}}\right)=\beta(\psi(d(1,2))) \psi(d(1,2)) .
$$

Therefore, we can not apply the Mizogochi-Takahashi type contraction [22].

Corollary 3. Let $f$ be self-mapping on a complete metric space $(X, d)$. Given $\alpha: X^{2} \rightarrow[0, \infty)$, Let

(i) $f$ is triangular $\alpha$-admissible;

(ii) for all $\omega, \Omega \in X$ with $1 \leq \alpha(\omega, \Omega)$ and $d(f \omega, f \Omega)>0$,

$$
d(f \omega, f \Omega) \leq \frac{\beta(d(\omega, \Omega)) d(\omega, \Omega)}{\left(\sqrt{\beta(d(\omega, \Omega))}+\sqrt{d(\omega, \Omega)}-\sqrt{d(\omega, \Omega) \beta(d(\omega, \Omega))^{2}}\right.} ;
$$

(iii) there is $\varsigma_{0} \in X$ so that $\alpha\left(\varsigma_{0}, f \varsigma_{0}\right) \geq 1$;

(iv) either $f$ is continuous, or $(K)$ holds.

Then $f$ has a fixed point. Moreover, such a fixed point is unique, provided that $\alpha(r, s) \geq 1$ for all $r, s \in$ Fix $(f)$.

Proof. It suffices to take $F(t)=-\frac{1}{\sqrt{t}}+1$ and $\psi(t)=t$ in Theorem 3 and to use the fact $d(\omega, \Omega) \leq$ $M(\omega, \Omega)$.

Corollary 4. Let $f$ be self-mapping on a complete ordered metric space $(X, d, \preceq)$. Assume that

(i) for all $\omega, \Omega \in X$ with $\omega \preceq \Omega$ and $d(f \omega, f \Omega)>0$,

$$
d(f \omega, f \Omega) \leq \frac{\beta(d(\omega, \Omega)) d(\omega, \Omega)}{\left(\sqrt{\beta(d(\omega, \Omega))}+\sqrt{d(\omega, \Omega)}-\sqrt{d(\omega, \Omega) \beta(d(\omega, \Omega))^{2}}\right.}
$$

where $\beta \in \Phi$;

(ii) there is $\varsigma_{0} \in X$ such that $\varsigma_{0} \preceq f \varsigma_{0}$;

(iii) either $f$ is continuous, or $(H)$ holds.

Then $f$ has a fixed point. Moreover, if any two fixed points of $f$ are comparable, then such a fixed point is unique. 
Proof. It follows by taking $F(t)=-\frac{1}{\sqrt{t}}+1$ and $\psi(t)=t$ in Theorem 4 and using the inequality $d(\omega, \Omega) \leq M(\omega, \Omega)$

\section{Application}

Take $I=[0, T](T>0)$. Let $X=C(I, \mathbb{R})$ be the set of valued continuous functions defined on $I$. Consider

$$
d(\omega, \Omega)=\sup _{q \in I}(|\omega(q)-\Omega(q)|)=\|\omega-\Omega\|_{\infty}
$$

which is a metric on $X$. We endow on $X$ the partial order

$$
r \preceq s \Longleftrightarrow r(q) \leq s(q) \text {, for any } q \in I \text {. }
$$

We will resolve the following boundary value problem

$$
r^{\prime}(q)=f(q, r(q)), \quad q \in[0, T], \quad r(0)=r(T),
$$

where $f: I \times \mathbb{R} \rightarrow \mathbb{R}$ is continuous.

Theorem 5. Assume that there is $\mu>0$ such that for all $r, s \in X=C(I, \mathbb{R})$ with $r \preceq s$, we have

$$
\mid f(p, r(p))+\mu r(p)-f(p, s(p))-\mu s(p)) \mid \leq \frac{\mu|r(p)-s(p)|}{\left[1+\|r-s\|_{\infty}^{\frac{1}{2}}\left(e^{\mid \frac{\|r-s \mid\|_{\infty}}{2}}-1\right)\right]^{2}} ;
$$

for each $p \in[0, T]$. Then (27) has a solution in $C(I, \mathbb{R})$.

Proof. First, Equation (27) is equivalent to the linear first-order equation

$$
r^{\prime}(q)+\mu r(q)=F(q, r(q)), \quad q \in[0, T], \quad r(0)=r(T),
$$

where $F(q, r(q))=f(q, r(q))+\mu r(q)$. Also, the function $q \rightarrow F(q, r(q))$ is continuous. From (29), we have

$$
r(q)=r(0) e^{-\mu q}+\int_{0}^{T} e^{\mu(p-q)} F(p, r(p)) d p, \quad q \in[0, T] .
$$

Choose $q=T$ to have

$$
r(T)=r(0) e^{-\mu T}+\int_{0}^{T} e^{\mu(p-T)} F(p, r(p)) d p .
$$

Since $r(0)=r(T)$, we get

$$
r(0)=\frac{1}{e^{\mu T}-1} \int_{0}^{T} e^{\mu p} F(p, r(p)) d p .
$$

Substituting in (30), we obtain

$$
r(q)=\int_{0}^{T} G(q, p) F(p, r(p)) d p, q \in[0, T]
$$

where

$$
G(q, p)= \begin{cases}\frac{e^{\mu(T+p-q)}}{e^{\mu T}-1}, \quad 0 \leq p \leq q \leq T \\ \frac{e^{\mu(p-q)}}{e^{\mu T}-1}, \quad 0 \leq q \leq p \leq T\end{cases}
$$

Take $f: C(I, \mathbb{R}) \rightarrow C(I, \mathbb{R})$ as

$$
f r(q)=\int_{0}^{T} G(q, p) F(p, r(p)) d p, \quad q \in[0, T] .
$$


Now we show that $\int_{0}^{T} G(q, p) d p=\frac{1}{\mu}$. To see this, we have

$$
\begin{aligned}
\int_{0}^{T} G(q, p) d p & =\int_{0}^{q} \frac{e^{\mu(T+p-q)}}{e^{\mu T}-1} d p+\int_{q}^{T} \frac{e^{\mu(p-q)}}{e^{\mu T}-1} d p \\
& =\left.\frac{e^{\mu(T+p-q)}}{\mu\left(e^{\mu T}-1\right)}\right|_{0} ^{q}+\left.\frac{\left.e^{\mu(p-q)}\right)}{\mu\left(e^{\mu T}-1\right)}\right|_{q} ^{T} \\
& =\frac{e^{\mu(T)}-e^{\mu(T-q)}}{\mu\left(e^{\mu T}-1\right)}+\frac{e^{\mu(T-q)}-1}{\mu\left(e^{\mu T}-1\right)}=\frac{1}{\mu} .
\end{aligned}
$$

From [24], $f$ is nondecreasing and there is $\varsigma_{0} \in X$ so that $\varsigma_{0} \preceq f \varsigma_{0}$. Letting $r, s \in X$ (with $r \preceq s$ ) and using (28), we have for every $q \in[0, T]$,

$$
\begin{aligned}
|f r(q)-f s(q)| & =\mid \int_{0}^{T} G(q, p)(F(p, r(p))-F(p, s(p)) d p \mid \\
& \leq \int_{0}^{T} G(q, p) \mid F(p, r(p))-F(p, s(p) \mid d p \\
& =\int_{0}^{T} G(q, p)|f(p, r(p))+\mu r(p)-f(p, s(p))-\mu s(p)| d p \\
& \leq \int_{0}^{T} G(q, p) \frac{\mu|r(p)-s(p)|}{\left[1+\|r-s\|_{\infty}^{\frac{1}{2}}\left(e^{\frac{\| r-s}{2} \|_{\infty}}-1\right)\right]^{2}} d p \\
& \leq \frac{\mu\|r-s\|_{\infty}}{\left[1+\|r-s\|_{\infty}^{\frac{1}{2}}\left(e^{\frac{\|r-s\|_{\infty}}{2}}-1\right)\right]^{2}}\left(\int_{0}^{T} G(q, p) d p\right) \\
& =\frac{\|r-s\|_{\infty}}{\left[1+\|r-s\|_{\infty}^{\frac{1}{2}}\left(e^{\frac{\|r-s\|_{\infty}}{2}}-1\right)\right]^{2}} \\
& =\frac{\|r-s\|_{\infty}}{\left[1+\|r-s\|_{\infty}^{\frac{1}{2}}\left(e^{\frac{\|r-s\|_{\infty}}{2}}-1\right)\right]^{2}} .
\end{aligned}
$$

Taking the supremum to find that

$$
\begin{aligned}
d(f r, f s) & =\|f r-f s\|_{\infty} \\
& \leq \frac{\|r-s\|_{\infty}}{\left[1+\|r-s\|_{\infty}^{\frac{1}{2}}\left(e^{\frac{\|r-s\|_{\infty}}{2}}-1\right)\right]^{2}} \\
& =\frac{e^{-\|r-s\|_{\infty}\|r-s\|_{\infty}}}{\left[e^{-\frac{\|r-s\|_{\infty}}{2}}+\|r-s\|_{\infty}^{\frac{1}{2}}-\|r-s\|_{\infty}^{\frac{1}{2}} e^{-\|r-s\|_{\infty}}\right]^{2}} \\
& =\frac{\beta(d(r, s)) d(r, s)}{(\sqrt{\beta(d(r, s))}+\sqrt{d(r, s)}-\sqrt{d(r, s) \beta(d(r, s))})^{2}}
\end{aligned}
$$

where $\beta(t)=e^{-t}$. Therefore, by Corollary $4, f$ has a fixed point. Hence, there is a solution for (31) (and so for (27)).

Author Contributions: B.M. analyzed and prepared/edited the manuscript, V.P. analyzed and prepared the manuscript, H.A. analyzed and prepared/edited the manuscript, H.I. analyzed and prepared the manuscript. All authors read and approved the final manuscript.

Funding: This research received no external funding.

Acknowledgments: The authors are thankful to the anonymous referees for their useful and critical remarks on the paper.

Conflicts of Interest: The authors declare that they have no conflict of interest. 


\section{References}

1. Ran, A.C.M.; Reurings, M.C.B. A fixed point theorem in partially ordered metric sets and some applications to matrix equations. Proc. Amer. Math. Soc. 2003, 132, 1435-1443. [CrossRef]

2. Amini-Harandi A.; Emami, H. A fixed point theorem for contraction type maps in partially ordered metric spaces and application to ordinary diferential equations. Nonlinear Anal. 2010, 72, 2238-2242. [CrossRef]

3. Aydi, H.; Nashine, H.K.; Samet B.; Yazidi, H. Coincidence and common fixed point results in partially ordered cone metric spaces and applications to integral equations. Nonlinear Anal. 2011, 74, 6814-6825. [CrossRef]

4. Aydi, H.; Damjanovic, B.; Samet B.; Shatanawi, W. Coupled fixed point theorems for nonlinear contractions in partially ordered G-metric spaces. Math. Comput. Model. 2011, 54, 2443-2450. [CrossRef]

5. Aydi, H. On common fixed point theorems for $(\psi, \varphi)$-generalized $f$-weakly contractive mappings. Miskolc Math. Notes 2013, 14, 19-30. [CrossRef]

6. Aydi, H.; Karapinar, E.; Mustafa, Z. Coupled coincidence point results on generalized distance in ordered cone metric spaces. Positivity 2013, 17, 979-993. [CrossRef]

7. Işık, H.; Turkoglu, D. Fixed point theorems for weakly contractive mappings in partially ordered metric-like spaces. Fixed Point Theory Appl. 2013, 2013, 51. [CrossRef]

8. Işık, H.; Radenovic, S. A new version of coupled fixed point results in ordered metric spaces with applications. U.P.B. Sci. Bull. Series A 2017, 79, 131-138.

9. Işık, H.; Turkoglu, D. Some fixed point theorems in ordered partial metric spaces. J. Inequal. Spec. Funct. 2013, 4, 13-18.

10. Işık, H.; Ionescu, C. New type of multi-valued contractions with related results and applications. U.P.B. Sci. Bull. Series A 2018, 80, 13-22.

11. Moradi, S.; Karapinar, E.; Aydi, H. Existence of solutions for a periodic boundary value problem via generalized weakly contractions. Abstr. Appl. Anal. 2013. [CrossRef]

12. Nieto, J.J.; Rodríguez-López, R. Contractive mapping theorems in partially ordered sets and applications to ordinary differential equations. Order 2005, 22, 223-239. [CrossRef]

13. Nieto J.J.; Rodríguez-López, R. Existence and uniqueness of fixed point in partially ordered sets and applications to ordinary differential equations. Acta Math. Sin. 2007, 23, 2205-2212. [CrossRef]

14. Zaslavski, A.J. Two fixed point results for a class of mappings of contractive type. J. Nonlinear Var. Anal. 2018, 2, 113-119.

15. Nadler, S.B. multivalued contraction mappings. Pacific J. Math. 1969, 30, 475-88. [CrossRef]

16. Aydi, H.; Abbas M.; Vetro, C. Common fixed points for multi-valued generalized contractions on partial metric spaces. RACSAM-Revista de la Real Academia de Ciencias Exactas, Fsicas y Naturales. Serie A. Matematicas 2014, 108, 483-501. Available online: https:/ / link.springer.com/article/10.1007/s13398-013-0120-z (accessed on 6 May 2019). [CrossRef]

17. Aydi, H.; Felhi, A.; Karapinar E.; Sahmim, S. A Nadler-type fixed point theorem in dislocated spaces and applications. Miskolc Math. Notes 2018, 19, 111-124. [CrossRef]

18. Klim, D.; Wardowski, D. Fixed point theorems for set-valued contractions in complete metric spaces. J. Math. Anal. Appl. 2007, 334, 132-139. [CrossRef]

19. Petrusel, A.; Petrusel, G. On Reich's strict fixed point theorem for multi-valued operators in complete metric spaces. J. Nonlinear Var. Anal. 2018, 2, 103-112.

20. Reich, S. Fixed points of contractive functions. Boll. Unione Mat. Ital. 1972, 4, 26-42.

21. Mizoguchi, N.; Takahashi, W. Fixed point theorems for multi-valued mappings on complete metric space. J. Math. Anal. Appl. 1989, 141, 177-188. [CrossRef]

22. Gordji, M.E.; Ramezani, M. A generalization of Mizoguchi and Takahashi's theorem for single-valued mappings in partially ordered metric spaces. Nonlinear Anal. 2011, 74, 4544-4549. [CrossRef] 
23. Karapınar, E.; Kumam, P.; Salimi, P. On $\alpha-\psi$-Meir-Keeler contractive mappings. Fixed Point Theory Appl. 2013, 2013, 94. [CrossRef]

24. Harjani, J.; López B.; Sadarangani, K. Fixed point Theorems for mixed monotone operators and applications to integral equations. Nonlinear Anal. 2011, 74, 1749-1760. [CrossRef]

(C) 2019 by the authors. Licensee MDPI, Basel, Switzerland. This article is an open access article distributed under the terms and conditions of the Creative Commons Attribution (CC BY) license (http://creativecommons.org/licenses/by/4.0/). 